\title{
Fungal Keratitis and Ocular Surface Squamous Neoplasia in a HIV Positive Patient
}

\section{Thanuja Pradeep, Ramya Marappa}

M. S. Ramaiah Medical College, Bengaluru, India

Email: thanugopal@yahoo.co.in,ramyaamgl@yahoo.co.in

Received 18 March 2016; accepted 9 May 2016; published 12 May 2016

Copyright (C 2016 by authors and Scientific Research Publishing Inc.

This work is licensed under the Creative Commons Attribution International License (CC BY). http://creativecommons.org/licenses/by/4.0/

\section{(c) (i) Open Access}

\begin{abstract}
Aim: We report a case of fungal keratitis and Ocular Surface Squalous Neoplasia (OSSN) occurring in a HIV positive patient. Method: A 32-year-old female patient presented with a non-healing ulcer, which was diagnosed as fungal keratitis. On examination her ocular surface also revealed OSSN on the nasal bulbar conjunctiva. Serological investigations revealed HIV positive status with CD 4 counts of 61 cells/ $\mu$ l. Patient was treated for fungal keratitis with topical antifungals and also underwent excision biopsy of OSSN which was reported as conjunctival intraepithelial neoplasia. The ulcer showed significant response to topical medication only after the commencement of Anti-Retroviral Therapy (ART). Conclusion: This case emphasizes on the importance of a thorough ocular examination to rule out associated ocular conditions and investigations to rule out immunosuppressive status in a non-healing ulcer.
\end{abstract}

Keywords

Fungal Keratitis, OSSN, HIV

\section{Introduction}

Immunosuppressed conditions associated with Ocular Surface Squamous Neoplasia (OSSN) has been emphasized in many previous publications especially in young individuals [1]-[3]. The risk factors for OSSN have been enumerated as UV exposure, smoking, exposure to petroleum products, but in young individuals below the age of 40 years HIV infection has been shown to be a major risk factor and associated with an aggressive disease [3].

Fungal keratitis is more common in tropical climate and there is an increased risk of occurrence in vegetative 
matter injury, diabetics, use of topical steroids and immunosuppressed conditions. Fungal keratitis has also been reported to occur at increased frequency in retroviral positive patients [4].

We present a case of a young patient presenting with both fungal keratitis and OSSN. On investigation the patient was detected to be HIV-positive with low CD 4 counts. To our knowledge a combined presentation has not been reported earlier in literature. This case reiterates the importance of complete ocular examination and the need to investigate for immunosuppression in cases of non-healing ulcers and OSSN, especially in young individuals.

\section{Case Report}

A 32-year-old female presented with pain in the left eye since 6 months which was on and off for which she was treated with topical drops (? corticosteroids). Since 2 months the pain had become continuous and not responding to any medication. Patient had a history of stick injury 5 years back for which she was treated and had no consequences due to it and had good vision following it. On examination her right eye examination was normal with vision 6/6 and anterior and posterior segment examination were normal. Left eye revealed vision of Counting fingers $1 \mathrm{~m}$ and a central $6 \times 7 \mathrm{~mm}$ ulcer was noted which had finger like projections with some deep and superficial vascularization probably related to old healed keratitis. She had a hypopyon measuring $1 \mathrm{~mm}$. Corneal scrapings sent on the first day showed $\mathrm{KOH}$ positive and gram stain negative and a diagnosis of Fungal keratitis was made. The patient also had a fleshy diffuse mass on the nasal conjunctiva with conjunctival rossettes, the mass was extending from caruncle to the nasal limbus and $1 \mathrm{~mm}$ into the cornea measuring about $10 \mathrm{~mm} \times$ $10 \mathrm{~mm}$ in size. The thickness of the mass was variable from $1 \mathrm{~mm}$ to $4 \mathrm{~mm}$ across the surface. The mass had variable pigmentation with patchy hyperpigmented areas. There were prominent feeder vessels. A diagnosis of OSSN was made (Figure 1). Blood investigations were sent for HIV and HBS Ag and liver function tests were done as she was considered for itraconazole commencement.

The investigation reports were as follows: HIV 1\&2-positive by immunoassay. Hemoglobin $-7 \mathrm{~g} / \mathrm{dl}$, Total leucocyte count (TLC)—4610 cells/cumm, N/L/E/M/B —67/22/03/8/0, ESR—140, RBC—2.22 m/cumm, PCV— 23.4\%, Peripheral smear-microcytic hypochromic anemia.

Liver function tests were deranged: Serum (Se). bilirubin $-0.21 \mathrm{mg} / \mathrm{dl}$, se. Direct bilirubin $-0.13 \mathrm{mg} / \mathrm{dl}$, Se total protein-8.7 g/dl, Se albumin—2.7 g/dl, A/G ratio-0.4 (N-1.2 - 1.5) Se aspartate transaminase (AST) $120 \mathrm{U} / \mathrm{L}\left(\mathrm{N} \_0\right.$ - 31), Se Alanine transaminase (ALT)—45 U/l, Se Alkanine phosphatase $722 \mathrm{U} / \mathrm{L}$ (N-35 104), Se gamma glutamyl transferase (GGT) — 261 IU/L (N—6 - 40), HBs Ag by VITROS ECi immunodiagnositic system-negative, CD 4 counts-61 cells/microl.

Ultrasound abdomen showed fatty hepatomegaly and splenomegaly, Sputum for AFB was negative. An internal medicine reference was taken for her systemic condition.

She was started on the first day with topical Natamycin 5\% drops hourly along with moxifloxacin $0.5 \%$ TID and homatropine TID. The ulcer responded to the topical medication initially with slight reduction in ulcer size and disappearance of hypopyon on the third day. She also underwent an excision biopsy for the OSSN on the fifth day, following the standard precautions of no-touch technique and intra-operative mitomycin $\mathrm{C}$ was applied and cryotherapy for the conjunctival margins. The HPE report showed features of invasive squamous cell carcinoma with conjunctival intraepithelial neoplasia with all margins free of the tumor (Figure 2). In view of a non-healing ulcer we did not consider for any post op mitomycin C. The ulcer after an initial improvement became unresponsive and remained status quo.

The patient was started on Anti-retroviral therapy (ART) on the seventh day. She was continued on the topical medications. After 3 days of ART patient showed good improvement. The ulcer responded to topical medications. Her ulcer healed and there were no signs of OSSN recurrence on 2 months follow-up (Figure 3).

\section{Discussion}

Our patient presented to us with a non-healing ulcer. Patient had irritation of the eye since 6 months and she was treated with topical corticosteroids and developed fungal keratitis. After the occurrence of fungal keratitis the treating ophthalmologist directed the treatment towards the ulcer and failed to recognize OSSN. She was referred to us in this stage as a non-healing ulcer. Misdiagnosis of OSSN as a benign surface disorder led to erroneous use of topical steroids which probably resulted in fungal keratitis.

The presence of non-healing fungal keratitis along with OSSN warranted a need for investigation for an im- 




Figure 1. OSSN on nasal conjunctiva and central corneal ulcer.

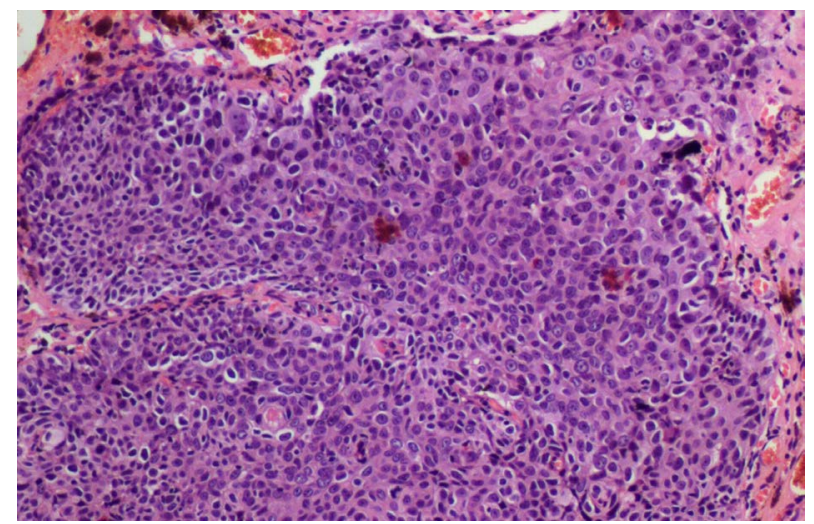

Figure 2. Histopathology section showing proliferation of dysplastic squamous cells.

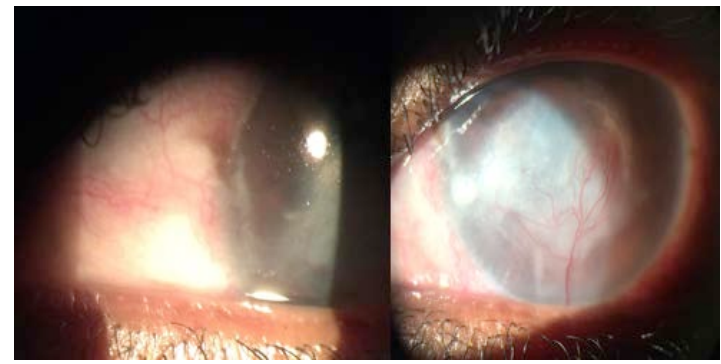

Figure 3. Healed corneal ulcer and resolved OSSN.

munosuppression in this case. HIV reports were positive thus emphasizing the fact that such cases need a systemic evaluation to identify the cause of non-responsiveness of an ulcer to topical medication.

The association of OSSN in HIV positive patients has been reported earlier especially in young individuals below 40 years [1]-[3]. MSelle $\mathrm{J}$ has reported increased risk of fungal keratitis in HIV positive patients in SubSaharan countries [4].

In HIV positive individuals with fungal keratitis, along with topical medications, patients need to be started on ART to see a significant clinical response. Presence of OSSN warrants an excision biopsy conforming to the standard treatment protocol. There has been a report of treating OSSN [5] with only ART but most studies support the importance of excision biopsy [6].

\section{Conclusion}

This case emphasizes the need for a thorough examination in cases of non-healing ulcer, both systemic and ocular. A high index of suspicion is required to identify immunosuppressed status. These cases show good response to topical treatment once they are started on antiretroviral therapy.

\section{References}

[1] Nagaiah, G., Stotler, C., Orem, J., Mwanda, W.O. and Remick, S.C. (2010) Ocular Surface Squamous Neoplasia in Pa- 
tients with HIV Infection in Sub-Saharan Africa. Current Opinion in Oncology, 22, 437-442. http://dx.doi.org/10.1097/CCO.0b013e32833cfcf9

[2] Pradeep, T.G., et al. (2012) Prevalence of Undiagnosed HIV Infection in Patients with Ocular Surface Squamous Neoplasia in a Tertiary Center in Karnataka, South India. Cornea, 31, 1282-1284. http://dx.doi.org/10.1097/ICO.0b013e3182479aed

[3] Weinstein, J.E. and Karp, C.L. (2013) Ocular Surface Neoplasias and Human Immunodeficiency Virus Infection. Current Opinion in Infectious Diseases, 26, 58-65. http://dx.doi.org/10.1097/QCO.0b013e32835b5969

[4] Mselle, J. (1999) Fungal Keratitis as an Indicator of HIV Infection in Africa. Tropical Doctor, 29, 133-135.

[5] Holkar, S., Mudhar, H.S., Jain, A., et al. (2005) Regression of Invasive Conjunctival Squamous Carcinoma in an HIVPositive Patient on Antiretroviral Therapy. International Journal of STD \& AIDS, 16, 782-783. http://dx.doi.org/10.1258/095646205774988028

[6] Mittal, R., Rath, S. and Vemuganti, G.K. (2013) Ocular Surface Squamous Neoplasia-Review of Etio-Pathogenesis and an Update on Clinico-Pathological Diagnosis. Saudi Journal of Ophthalmology, 27, 177-186. http://dx.doi.org/10.1016/j.sjopt.2013.07.002 